\title{
Ciclo de mejora en el aula en la materia de Legislación Farmacéutica: interpretando el modelo de Farmacia en España.
}

\section{Improvement Cycle in \\ Classroom in the matter of Pharmaceutical Law: interpreting the Pharmacy model in Spain.}

ESTEBAN MORENO TORAL

Orcid: https://orcid.org/0000-0002-3355-4128.

Universidad de Sevilla.

Departamento de Farmacia y

Tecnología Farmacéutica.

toral@us.es

DOI: http://dx.doi.org/10.12795/9788447231003.050

Pp.: 1044-1066 


\section{Descripción del contexto}

Iniciamos este Ciclo de Mejora en el Aula (CIMA) con un objetivo fundamental: optimizar el perfil crítico de la docencia que se imparte en la asignatura de Legislación Farmacéutica del 5o curso del Grado en Farmacia de la Universidad de Sevilla. De esta manera se pretende que sea el estudiante el protagonista de la actividad y que vaya alcanzando los conocimientos necesarios por sí mismo, trabajando los contenidos y reflexionando sobre estos. La asignatura donde realizamos la mejora docente es de carácter obligatorio y se imparte durante el primer cuatrimestre del curso académico. Tiene una gran vinculación en el desarrollo profesional que van a realizar los estudiantes en su futuro y por tanto los contenidos impartidos son bastante atractivos para los mismos. En España hay unas 22.100 oficinas de farmacia. Estos establecimientos vienen regulados legalmente por una serie de normas jurídicas. El modelo de farmacia español, conocido como mediterráneo, limita la propiedad de forma exclusiva a los farmacéuticos y además establece unos requisitos objetivos basados en criterios demográficos (ratio de habitantes por farmacia) y criterios geográficos (distancias mínimas a otras oficinas de farmacia y centros sanitarios públicos). Además, los procedimientos administrativos son complejos y con características particulares. A pesar de estas restricciones, el 99\% de la población española tienen este servicio farmacéutico a menos de diez minutos de su domicilio.

En varias ocasiones he participado en proyectos de innovación y mejora docente de la Universidad de Sevilla, y es por ello que cuento con cierta experiencia en la mejora de la docencia. Fruto de aquellas innovaciones he ido mejorando la actividad lo que se ha visto reflejado en los resultados docentes y en las encuestas de evaluación de los estudiantes. Pero siempre es posible dar un paso más para seguir mejorando (Bain, 2007).

Ciclos de Mejora en el Aula (2020). Experiencias de Innovación Docente de la US Esta obra se distribuye con la licencia Creative Commons 


\section{Diseño previo del CIMA}

\section{Mapa de Contenidos.}

En el desarrollo de la actividad se pretende que los estudiantes puedan responder con amplio conocimiento a las preguntas planteadas en cada sesión. Usamos una herramienta que lo posibilita que es la docencia invertida. Ello plantea un trabajo previo al aula, de carácter procedimental intelectual y actitudinal. Se trata de que busquen la información en las fuentes usando diferentes procedimientos y que las valoren.

En nuestro mapa de contenidos hemos representado las preguntas/problemas de cada sesión con cartelas de color blanco. Usamos rectángulos para los conceptos (color verde) y datos (color celeste). Para los procedimientos intelectuales usamos elipses de color rojo y para los procedimientos mecánicos círculos de color amarillo. Finalmente hemos utilizado rombos naranjas para los contenidos actitudinales.

Ciclos de Mejora en el Aula (2020). Experiencias de Innovación Docente de la US Esta obra se distribuye con la licencia Creative Commons 


\section{ESTEBAN MORENO TORAL}

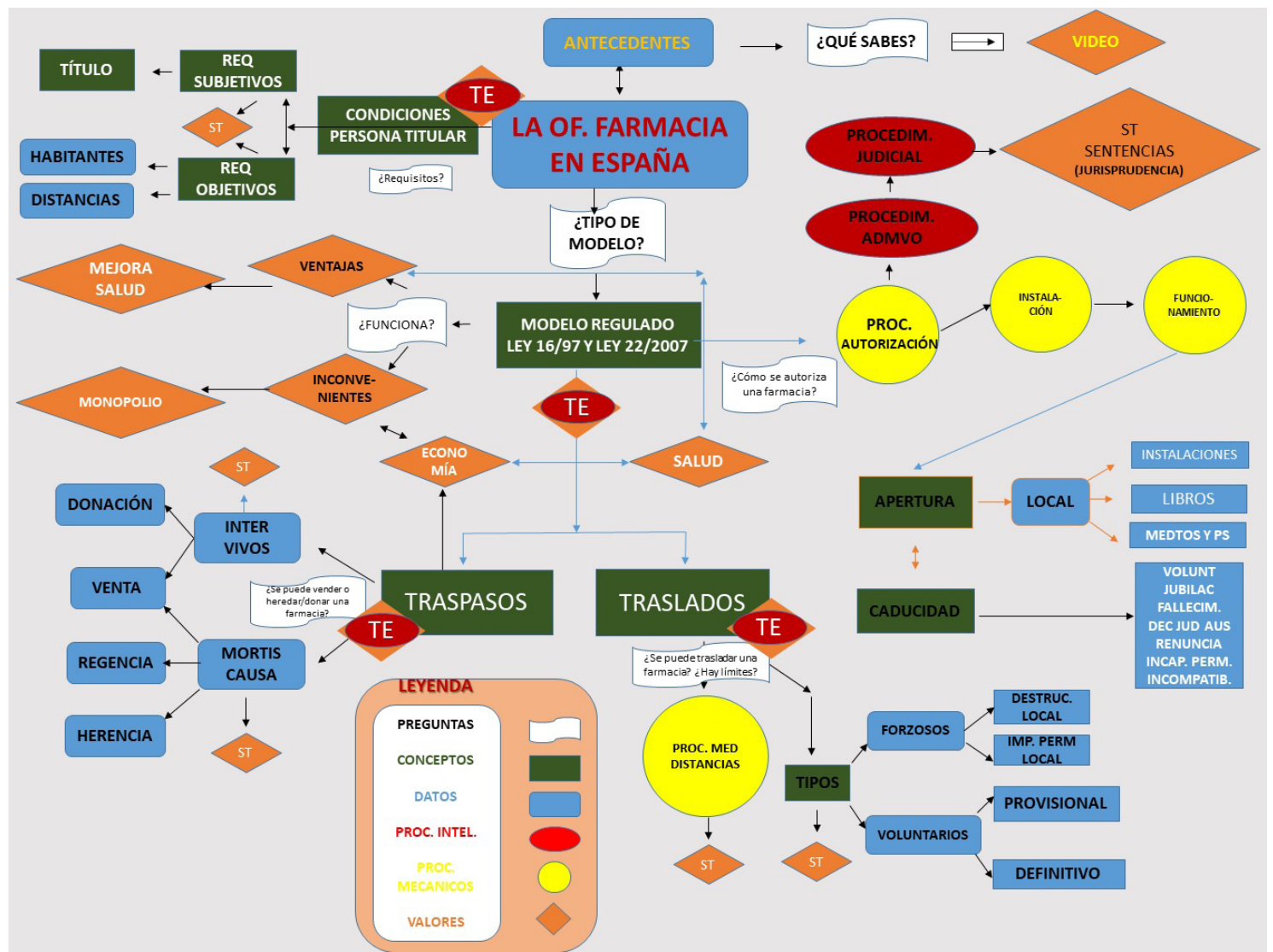

Figura 1. Esquema mapa de contenido propuesto

Ciclos de Mejora en el Aula (2020). Experiencias de Innovación Docente de la US (c) E) Esta obra se distribuye con la licencia Creative Commons (c) $(1) \bigoplus$ Reconocimiento-NoComercial-SinObraDerivada Internacional (CC BY-NC-ND 4.0.) 
Las preguntas-problemas (en cartelas blancas) son las que se van a plantear en el cuestionario, inicial y final, y responden a lo que el estudiante conoce antes de la actividad y lo que sabrá al finalizar la misma.

\section{Modelo metodológico básico propuesto y secuencia de actividades.}

El modelo metodológico se ha basado en la resolución de problemas y sobre todo en técnicas próximas a la línea de "dar clase con la boca cerrada" (Finkel, 2008). La intención es promover la participación estudiantil y que sea el profesor el que haga de catalizador cuando se llegue a vías muertas en el aula.

Cada modelo se plantea en 4 sesiones de dos horas cada una. En cada sesión se sigue un modelo que se va a repetir en cada una de ellas. Previamente se divide el grupo de 60 estudiantes en 4 grandes grupos de 15. A su vez dentro de estos grupos de 15 estudiantes de establecen tres nuevos subgrupos ( $a, b$ y c) de 5 miembros cada uno que van a trabajar las diferentes materias de cada sesión (dos ciclos IE / AC por sesión). De este modo el total de 60 estudiantes han quedado divididos en 12 subgrupos (3 por cada sesión). Cada sesión sigue este modelo:

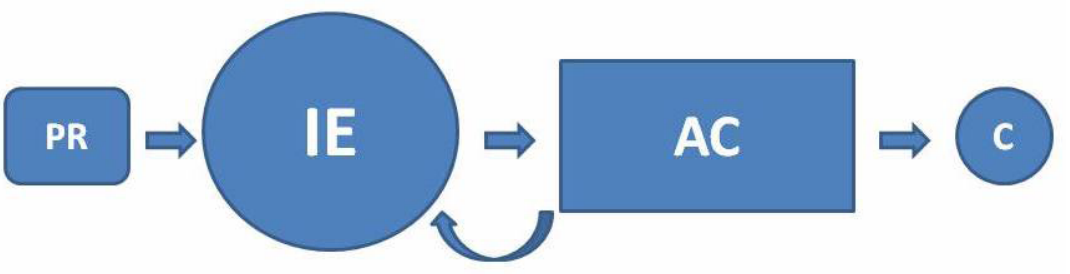

Figura 2. Modelo metodológico seguido

PR: planteamiento del problema. El profesor plantea la temática a trabajar en cada sesión y las preguntas/problemas (5/10 minutos). IE: Ideas de los estudiantes. De manera previa se han repartido las temáticas de cada sesión que deben haber preparado con anterioridad en subgrupos de 5 estudiantes. Toma de conciencia, trabajo y presentación

Ciclos de Mejora en el Aula (2020). Experiencias de Innovación Docente de la US Esta obra se distribuye con la licencia Creative Commons 
por los estudiantes del tema. Se aportan datos objetivos y aspectos subjetivos o de opinión (45/50 minutos). AC: Actividad de Contraste. El profesor matiza, contrasta y aclara las ideas expuestas (10 minutos). Se procede a un debate entre los estudiantes sobre cada temática (20 minutos). IE: Exposición por los estudiantes de otro tema o una sentencia judicial sobre la temática que ha generado jurisprudencia (10 minutos). AC: Aclaración sobre la materia/ sentencia y debate (10 minutos). C: Conclusiones y plasmación de lo aprendido (5 minutos).

La secuencia de actividades que hemos planteado ha sido en coherencia con el modelo, indicando cada actividad a qué fase del mismo corresponde. Se parte de dos horas por sesión, tomando un total de 115 minutos. Las actividades en grupo, que los estudiantes desarrollan previas a las sesiones en clase, pretenden que estos adquirieran unos conocimientos procedimentales de datos así como actitudinales de debate entre ellos. El objetivo final es que ellos mismos avancen en su escalera de conocimientos, nutriéndose tanto de forma autónoma como del aprendizaje de demás compañeros y del profesor. Con este trabajo previo fomentamos el desarrollo de los conocimientos conceptuales, procedimentales y en especial los actitudinales. Incorporar el debate y unas conclusiones finales nos permite la puesta en común de ideas. Pasamos a reflejar en tablas la secuencia de actividades.

Tabla 1. Actividades programadas en la sesión primera

\begin{tabular}{|c|c|c|c|}
\hline \multicolumn{4}{|c|}{ EL MODELO FARMACÉUTICO ESPAÑOL: VENTAJAS E INCONVENIENTES } \\
\hline ACTIVIDAD & INTERVIENE & DESCRIPCIÓN & TIEMPO \\
\hline Trabajo previo & Grupo 1 & Documentación en grupo & En casa \\
\hline Introducción & Profesor & $\begin{array}{c}\text { Descripción breve de la } \\
\text { historia de la legislación } \\
\text { farmacéutica española }\end{array}$ & 5 minutos \\
\cline { 2 - 4 } & Video & $\begin{array}{c}\text { Visionado de video del } \\
\text { modelo farmacéutico español }\end{array}$ & 4 minutos \\
\hline
\end{tabular}

Ciclos de Mejora en el Aula (2020). Experiencias de Innovación Docente de la US Esta obra se distribuye con la licencia Creative Commons 


\begin{tabular}{|c|c|c|c|}
\hline $\begin{array}{l}\text { Planteamiento } \\
\text { del problema }\end{array}$ & Preguntas & $\begin{array}{c}\text { a. ¿Qué sabes acerca de } \\
\text { la oficina de farmacia en } \\
\text { España? } \\
\text { b. ¿Funciona? }\end{array}$ & 1 minuto \\
\hline \multirow{3}{*}{$\begin{array}{l}\text { Ideas de los } \\
\text { estudiantes }\end{array}$} & 1.a & $\begin{array}{l}\text { Tipo de modelo. } \\
\text { Normas nacionales y } \\
\text { autonómicas. }\end{array}$ & 20 minutos \\
\hline & 1.b & Ventajas. Aspectos sanitarios & 15 minutos \\
\hline & 1.c & $\begin{array}{l}\text { Inconvenientes. Aspectos } \\
\text { económicos }\end{array}$ & 15 minutos \\
\hline \multirow{2}{*}{$\begin{array}{l}\text { Actividad de } \\
\text { Contraste }\end{array}$} & Video & $\begin{array}{l}\text { Visionado de video del } \\
\text { modelo anglosajón }\end{array}$ & 10 minutos \\
\hline & Debate & Confrontación de ideas & 20 minutos \\
\hline $\begin{array}{l}\text { Ideas } \\
\text { estudiantes }\end{array}$ & 1.a & Exposición sentencia UE & 10 minutos \\
\hline \multirow[b]{2}{*}{$\begin{array}{l}\text { Actividad de } \\
\text { Contraste }\end{array}$} & Profesor & Aspectos claves & 4 minutos \\
\hline & Debate & $\begin{array}{l}\text { Confrontación de modelos. } \\
\text { Intervenciones de dos } \\
\text { estudiantes de cada subgrupo } \\
\text { (un minuto máximo) }\end{array}$ & 6 minutos \\
\hline Conclusiones & Profesor & Cuestiones fundamentales & 5 minutos \\
\hline
\end{tabular}

Ciclos de Mejora en el Aula (2020). Experiencias de Innovación Docente de la US Esta obra se distribuye con la licencia Creative Commons 
Tabla 2. Actividades programadas en la sesión segunda

\begin{tabular}{|c|c|c|c|}
\hline \multicolumn{4}{|c|}{ REQUISITOS Y PROCEDIMIENTOS APERTURAS DE FARMACIAS } \\
\hline ACTIVIDAD & INTERVIENE & DESCRIPCIÓN & TIEMPO \\
\hline Trabajo previo & Grupo 2 & Documentación en grupo & En casa \\
\hline \multirow[t]{2}{*}{ Introducción } & \multirow[t]{2}{*}{ Profesor } & $\begin{array}{l}\text { Requisitos objetivos y } \\
\text { subjetivos }\end{array}$ & 2 minutos \\
\hline & & $\begin{array}{l}\text { Procedimientos } \\
\text { administrativos y } \\
\text { judiciales }\end{array}$ & 2 minutos \\
\hline $\begin{array}{l}\text { Planteamiento del } \\
\text { problema }\end{array}$ & Preguntas & $\begin{array}{l}\text { ¿En base a qué } 3 \text { criterios } \\
\text { se otorgan las aperturas? } \\
\text { ¿Cómo se autoriza una } \\
\text { farmacia? }\end{array}$ & 1 minuto \\
\hline \multirow[t]{3}{*}{$\begin{array}{l}\text { Ideas de los } \\
\text { estudiantes }\end{array}$} & 2.a & $\begin{array}{l}\text { Requisitos subjetivos. } \\
\text { Condiciones respecto a la } \\
\text { persona (8 requisitos) }\end{array}$ & 15 minutos \\
\hline & 2.b & $\begin{array}{l}\text { Criterios demográficos } \\
\text { (habitantes) }\end{array}$ & 15 minutos \\
\hline & 2.c & $\begin{array}{l}\text { Criterios geográficos } \\
\text { (distancias) }\end{array}$ & 15 minutos \\
\hline \multirow[t]{2}{*}{$\begin{array}{l}\text { Actividad de } \\
\text { Contraste }\end{array}$} & Profesor & $\begin{array}{l}\text { Aspectos esenciales } \\
\text { modelo }\end{array}$ & 5 minutos \\
\hline & Debate & Confrontación de ideas & 10 minutos \\
\hline \multirow[t]{3}{*}{$\begin{array}{l}\text { Ideas de los } \\
\text { estudiantes }\end{array}$} & 2.a & $\begin{array}{l}\text { Procedimiento } \\
\text { administrativo }\end{array}$ & 10 minutos \\
\hline & 2.b & Procedimiento judicial & 10 minutos \\
\hline & 2.c & $\begin{array}{l}\text { Instalación y } \\
\text { Funcionamiento }\end{array}$ & 10 minutos \\
\hline \multirow{2}{*}{$\begin{array}{l}\text { Actividad de } \\
\text { Contraste }\end{array}$} & 2.a & Exposición de Sentencia & 9 minutos \\
\hline & $\begin{array}{l}\text { Debate entre } \\
\text { 2.b y } 2 . c\end{array}$ & Confrontación de ideas & 6 minutos \\
\hline Conclusiones & Profesor & $\begin{array}{l}\text { Cuestiones } \\
\text { fundamentales }\end{array}$ & 5 minutos \\
\hline
\end{tabular}

Ciclos de Mejora en el Aula (2020). Experiencias de Innovación Docente de la US Esta obra se distribuye con la licencia Creative Commons 
Tabla 3. Actividades programadas en la sesión tercera

\begin{tabular}{|l|l|l|l|}
\hline \multicolumn{5}{|c|}{ TRASPASOS DE OFICINAS DE FARMACIA } \\
\hline ACTIVIDAD & \multicolumn{1}{|c|}{ INTERVIENE } & \multicolumn{1}{c|}{ DESCRIPCIÓN } & \multicolumn{1}{c|}{ TIEMPO } \\
\hline Trabajo previo & Grupo 3 & Documentación en grupo & En casa \\
\hline \multirow{2}{*}{ Introducción } & Profesor & $\begin{array}{l}\text { Traspasos de la propiedad- } \\
\text { titularidad de oficina de } \\
\text { farmacia }\end{array}$ & 4 minutos \\
\hline \multirow{2}{*}{$\begin{array}{l}\text { Planteamiento } \\
\text { del problema }\end{array}$} & Preguntas & $\begin{array}{l}\text { ¿Se puede vender? } \\
\text { ¿Se puede heredar/donar una } \\
\text { farmacia? }\end{array}$ & 1 minuto \\
\hline \multirow{2}{*}{$\begin{array}{l}\text { Ideas de los } \\
\text { estudiantes }\end{array}$} & $3 . a$ & Herencia Farmacia & 15 minutos \\
\cline { 2 - 5 } & $3 . b$ & Regencia & 15 minutos \\
\cline { 2 - 5 } & 3.c & Reserva titularidad por estudios & 15 minutos \\
\hline $\begin{array}{l}\text { Actividad de } \\
\text { Contraste }\end{array}$ & 3.a & Sentencia herencia farmacia & 10 minutos \\
\cline { 2 - 5 } & Debate & Confrontación de ideas & 10 minutos \\
\hline $\begin{array}{l}\text { Ideas de los } \\
\text { estudiantes }\end{array}$ & 3.a & Donación & 10 minutos \\
\cline { 2 - 5 } & 3.b & Venta & 15 minutos \\
\hline $\begin{array}{l}\text { Actividad de } \\
\text { Contraste }\end{array}$ & 3.c & Sentencia venta farmacia & 10 minutos \\
\cline { 2 - 5 } & Debate & Confrontación de ideas & 5 minutos \\
\hline Conclusiones & Profesor & Cuestiones fundamentales & 5 minutos \\
\hline
\end{tabular}

Tabla 4. Actividades programadas en la sesión cuarta

\begin{tabular}{|c|c|c|c|}
\hline \multicolumn{4}{|c|}{ TRASLADOS DE OFICINAS DE FARMACIA } \\
\hline ACTIVIDAD & INTERVIENE & DESCRIPCIÓN & TIEMPO \\
\hline Trabajo previo & Grupo 4 & Documentación en grupo & En casa \\
\hline Introducción & Profesor & $\begin{array}{l}\text { Traslados de la oficina de } \\
\text { farmacia }\end{array}$ & 4 minutos \\
\hline $\begin{array}{l}\text { Planteamiento } \\
\text { del problema }\end{array}$ & Preguntas & $\begin{array}{l}\text { ¿Se puede trasladar una } \\
\text { farmacia? } \\
\text { ¿Hay límites? }\end{array}$ & 1 minuto \\
\hline \multirow{3}{*}{$\begin{array}{l}\text { Ideas de los } \\
\text { estudiantes }\end{array}$} & 4. $\mathrm{a}$ & Traslados forzosos & 15 minutos \\
\hline & 4.b & $\begin{array}{l}\text { Traslados voluntarios } \\
\text { provisionales }\end{array}$ & 15 minutos \\
\hline & 4.c & Traslados voluntarios definitivos & 15 minutos \\
\hline
\end{tabular}

Ciclos de Mejora en el Aula (2020). Experiencias de Innovación Docente de la US Esta obra se distribuye con la licencia Creative Commons Reconocimiento-NoComercial-SinObraDerivada Internacional (CC BY-NC-ND 4.0.) 


\begin{tabular}{|l|l|l|l|}
\hline \multirow{2}{*}{$\begin{array}{l}\text { Actividad de } \\
\text { Contraste }\end{array}$} & 4.a & Sentencia traslado farmacia & 10 minutos \\
\cline { 2 - 4 } & Debate & Confrontación de ideas & 10 minutos \\
\hline $\begin{array}{l}\text { Ideas de los } \\
\text { estudiantes }\end{array}$ & $4 . \mathrm{b}$ & Medición de distancias & 10 minutos \\
\hline \multirow{2}{*}{$\begin{array}{l}\text { Actividad de } \\
\text { Contraste }\end{array}$} & $4 . c$ & $\begin{array}{l}\text { Sentencia medición distancias } \\
\text { entre farmacias }\end{array}$ & 10 minutos \\
\cline { 2 - 5 } & Debate & Confrontación de ideas & 10 minutos \\
\hline Conclusiones & Profesor & Cuestiones fundamentales & 5 minutos \\
\hline $\begin{array}{l}\text { Cuestionario } \\
\text { Final }\end{array}$ & Estudiantes & $\begin{array}{l}\text { Se contestan los 4 bloques de } \\
\text { preguntas }\end{array}$ & 5 minutos \\
\hline Despedida & Profesor & Valoración y agradecimiento & 5 minutos \\
\hline
\end{tabular}

\section{Cuestionario previo y final.}

Antes de realizar el CIMA en las 8 horas programadas se pasó un cuestionario inicial a los estudiantes. El objetivo primordial era evaluar el aprendizaje. Este cuestionario inicial lo realizaron 60 estudiantes. Se volvió a pasar al finalizar el CIMA. Se consiguió que la totalidad de los estudiantes contestara también el cuestionario final.

El cuestionario inicial que se les facilitó iba encaminado a descubrir cuáles eran sus esquemas mentales previos en relación con la temática y cómo relacionan la normativa con el funcionamiento real de las farmacias españolas. Es una materia, que aun siendo de naturaleza técnica, los conceptos básicos y el contenido de muchos aspectos son susceptibles de debate y opiniones encontradas.

Tanto el cuestionario previo como final se han hecho de forma anónima parcial. Para comparar estos cuestionarios inicial y final se propone a cada estudiante que identifique su cuestionario con 3 letras y un número. Primera letra de primer apellido, primera letra de segundo apellido, primera letra de nombre, y última cifra del DNI.

Se realizan una serie de preguntas divididas en 4 bloques o sesiones:

Ciclos de Mejora en el Aula (2020). Experiencias de Innovación Docente de la US Esta obra se distribuye con la licencia Creative Commons 
SESIÓN 1: ¿Qué sabes acerca de la oficina de farmacia en España? ¿Funciona?

SESIÓN 2: ¿En base a qué 3 criterios se otorgan las aperturas? ¿Cómo se autoriza una farmacia?

SESIÓN 3: ¿Se puede vender una farmacia? ¿Se puede heredar/donar una farmacia?

SESIÓN 4: ¿Se puede trasladar una farmacia? ¿Hay límites?

\section{Aplicación del CIMA}

\section{Relato resumido de las sesiones}

Martes 27 de octubre. Esta primera sesión va encaminada al planteamiento del problema/idea central, de forma que se contextualiza el tema y se intenta promover la curiosidad/interés de los estudiantes. El problema fundamental es debatir sobre el modelo de farmacia en España y las alternativas con sus pros y contras. Los contenidos que se van a tratar son esencialmente de carácter conceptos y datos, y sobre todo actitudinales. Estos últimos pretenden valorar críticamente los resultados del sistema.

Se inicia explicando los antecedentes del modelo existente (5 minutos). Se visiona seguidamente un video de unos 4 minutos en que se aborda el modelo farmacéutico español: https://www.youtube.com/watch?v=kNG4axgMSnA

A continuación se recuerdan las preguntas iniciales realizadas, como objetivo a resolver en esta sesión: ¿Qué sabes acerca de la oficina de farmacia en España? ¿Funciona?

Participan de forma directa tres subgrupos de 5 estudiantes cada uno denominados 1.a; 1.b y 1.c. Comienza el subgrupo 1.a exponiendo el tipo de modelo en España, llamado mediterráneo, con las normas nacionales y autonómicas que lo regulan haciendo especial hincapié en los

Ciclos de Mejora en el Aula (2020). Experiencias de Innovación Docente de la US Esta obra se distribuye con la licencia Creative Commons 
aspectos económicos y sanitarios (20 minutos). Seguidamente el subgrupo 1.b plantea las ventajas del modelo con especial incidencia en la mejora de la salud a la población (15 minutos). Se termina esta fase con la intervención del subgrupo 1.c con los inconvenientes del modelo recalcando cuestiones del monopolio y de la restricción a la libertad de establecimiento (15 minutos).

Como actividad de contraste se visiona un modelo totalmente contrario como es el anglosajón https://www. youtube.com/watch?v=RsksGYLuMrA (10 minutos), para dar paso a un debate de contraste entre ambos modelos con sus pros y contras, interviniendo los estudiantes de forma exclusiva (20 minutos). Se entabla una fuerte oposición ya que intervienen 3 estudiantes que consideran el modelo español contrario a normas liberalizadoras y por tanto incoherente. El profesor permanece callado. Solo actúa dinamizando el debate preguntando a otros estudiantes que aún no habían intervenido para que la implicación de todos sea masiva.

Una vez finalizado el debate, el grupo 1.a realiza la exposición de una sentencia judicial de 2010 sobre la libertad de establecimientos de farmacia en la Unión Europea. Se les proporciona un artículo http://dx.doi.org/10.4067/ S0718-68512010000200011 (10 minutos).

Como actividad de contraste el profesor matiza en 4 minutos las cuestiones clave y abre un nuevo debate con intervenciones de un minuto por estudiante (dos por cada subgrupo). Las opiniones siguen enfrentadas y todos escuchan los argumentos que defienden cada postura. Para terminar la primera sesión se establecen las conclusiones como reflejo de lo aprendido (5 minutos).

Jueves 29 de octubre. En la segunda sesión tratamos dos aspectos, por un lado, los requisitos generales de la normativa y por otro los procedimientos. En el primer bloque son contenidos de tipo conceptos y datos, mientras que en el segundo son básicamente de tipo procedimental tanto intelectual como mecánico. 
Se inicia explicando brevemente el profesor la materia a tratar. A continuación, se recuerdan las dos preguntas iniciales planteadas: ¿En base a qué 3 criterios se otorgan las aperturas? ¿Cómo se autoriza una farmacia? Participan 3 subgrupos de 5 estudiantes cada uno denominados 2.a; 2.b y 2.c.

Comienza el subgrupo 2.a exponiendo los requisitos subjetivos exigidos o condiciones respecto a la persona. Son un total de 8 requisitos, siendo el primero la exigencia exclusiva de tenencia del título de farmacéutico para ostentar la propiedad de una farmacia (15 minutos). Seguidamente el subgrupo 2.b desarrolla los criterios de ratios de habitantes por oficinas de farmacia (15 minutos). El último subgrupo, el 2.c, expone los requisitos geográficos o de distancias mínimas establecidos entre colindantes y con respecto a centros de salud pública (15 minutos).

Como actividad de contraste se repasan y matizan los aspectos esenciales del modelo por parte del profesor de forma breve (5 minutos), iniciando a continuación un debate de confrontación de ideas entre los tres grupos. También participan espontáneamente 4 compañeros (10 minutos).

A continuación, tiene lugar un nuevo ciclo de exposición de ideas con los aspectos formales relativos al procedimiento administrativo, judicial y los requisitos para la instalación y funcionamiento de la farmacia (10 minutos cada subgrupo). Como actividad de contraste se expone una sentencia para comprobar la aplicación real de los aspectos expuestos anteriormente (subgrupo 2.a) y se abre un nuevo debate con intervenciones de un minuto por estudiante. Para terminar la segunda sesión el profesor destaca en las conclusiones los aspectos más importantes (5 minutos).

Martes 3 de noviembre. En la tercera sesión se trabajó lo relativo al cambio de propiedad-titularidad de la oficina de farmacia. Puede ser de tipo mortis-causa o inter-vivos. 
En los cinco primeros minutos el profesor plantea la temática y las preguntas de referencia que abren la sesión. Las ideas de los estudiantes se plantean a continuación en tres turnos de 15 minutos abordando los aspectos relativos a los tipos mortis-causa, que son la herencia, la regencia y la reserva de titularidad por estudios a los herederos. Como actividad de contraste se plantea por el subgrupo 2.a una sentencia relativa a herencia de farmacia para conocer cuál es el criterio de los jueces y magistrados en España al respecto. Se completa con un debate entre todos los estudiantes para conocer los diferentes pareceres. Varios de los estudiantes manifiestan que es totalmente injusto que una farmacia adquirida en un concurso se pueda vender, donar o heredar. Lo ven incongruente. Una estudiante defiende que hay una inversión económica y por tanto un volumen de negocio y fidelización. Esta actividad de contraste tuvo unos 20 minutos en total.

Se inicia una nueva secuencia de ideas de los estudiantes con la exposición por ellos de los aspectos del cambio de propiedad-titularidad inter-vivos. Se aborda la donación y la venta, con 10 y 15 minutos respectivamente. Como actividad de contraste se expone una sentencia relativa a la compra-venta de farmacia con las implicaciones del Derecho Civil (10 minutos) y un debate breve de 5 minutos sobre la misma. Esta sesión resulta muy confrontada, hasta tensa en algunos momentos, por las diferentes opiniones.

Finalizamos la sesión con el resumen de las cuestiones fundamentales del traspaso de oficinas de farmacia. Los contenidos de esta sesión son fundamentalmente de conceptos y datos, aunque los contenidos actitudinales sirven de contraste con las sentencias que exponen el criterio del sistema judicial y los debates con la opinión de los estudiantes.

Jueves 5 de noviembre. La cuarta y última sesión trata los traslados de oficinas de farmacia. Al igual que en las

Ciclos de Mejora en el Aula (2020). Experiencias de Innovación Docente de la US Esta obra se distribuye con la licencia Creative Commons 
anteriores se inicia con una introducción y planteamiento del problema por parte del profesor (5 minutos).

El primer bloque de ideas de los estudiantes aborda los diferentes tipos de traslados. Se trata de contenidos de conceptos y datos y se abordan los traslados forzosos, los voluntarios provisionales y definitivos. Cada subgrupo cuenta con 15 minutos. Como actividad de contraste se expone una sentencia tipo que muestra el criterio de aplicación de las normas (10 minutos) junto a un debate estudiantil de 10 minutos sobre la misma (deben intervenir varios estudiantes de cada subgrupo, así como del resto en exposiciones de 1 minuto). El debate es más sosegado porque la materia no está tan polarizada como en el traspaso.

En el segundo bloque se estudian aspectos procedimentales tratando los criterios para medir las distancias mínimas (10 minutos) y como actividad de contraste se expone por los estudiantes una sentencia que versa sobre cómo medir estas distancias (10 minutos) y se abre un debate para contrastar opiniones (10 minutos).

Terminamos el tema de los traslados con las conclusiones por parte del profesor.

Para finalizar el CIMA se pasa a los estudiantes de nuevo el cuestionario de preguntas para que contesten y podamos comparar la escalera de aprendizaje adquirida en estas 4 sesiones de 8 horas en total. Se realiza una pequeña valoración de la experiencia por parte del profesor y se agradece la participación.

\section{Evaluación del aprendizaje de los estudiantes.}

Se han agrupado los resultados en 4 niveles llamados modelos A, B, C y D:

MODELO A: El nivel de conocimientos es mínimo, erróneo o nulo.

MODELO B: El nivel de conocimientos es medio, incompleto o con lagunas.

Ciclos de Mejora en el Aula (2020). Experiencias de Innovación Docente de la US Esta obra se distribuye con la licencia Creative Commons 
MODELO C: El nivel de conocimientos es alto pero sin concretar demasiado.

MODELO D: El nivel de conocimientos es elevado y desarrollado.

Seguidamente se han agrupado las respuestas a las preguntas de cada sesión, con el número de las mismas reunidas en los modelos, dadas al inicio y al final, incorporadas a tablas. Las escaleras de aprendizaje, inicial y final con los resultados, se han enfrentado en figuras para comprobar la evolución del estudiante, agrupados en los cuatro modelos.

Tabla 5. Respuestas a las preguntas: ¿Qué sabes acerca de la oficina de farmacia en España? ¿Funciona?

\begin{tabular}{|c|c|c|}
\hline NIVELES DE CONOCIMIENTO & $\begin{array}{c}\text { CUESTIONARIO } \\
\text { INICIAL }\end{array}$ & $\begin{array}{c}\text { CUESTIONARIO } \\
\text { FINAL }\end{array}$ \\
\hline $\begin{array}{c}\text { NIVEL A (Muy poco/Algo. Creo que no/ } \\
\text { que sí) }\end{array}$ & $36(60 \%)$ & $0(0 \%)$ \\
\hline NIVEL B (Algo/Creo que sí) & $15(25 \%)$ & $5(8,33 \%)$ \\
\hline NIVEL C (Bastante/Creo que no) & $3(5 \%)$ & $35(58,33 \%)$ \\
\hline NIVEL D (Bastante/Creo que sí) & $6(10 \%)$ & $20(33,33 \%)$ \\
\hline
\end{tabular}

Los resultados de la tabla 5 muestran en esta primera sesión una favorable evolución en el proceso de enseñanza-aprendizaje del estudiantado de la asignatura. Así en el cuestionario inicial el $60 \%$ de los mismos se situaban en el nivel $A$, por tanto sin ningún o con bajo conocimiento sobre los contenidos, y en el cuestionario final se reduce al $0 \%$. Lo más llamativo, es que en el cuestionario final más del $90 \%$ se encuentran en el nivel C/D, y por tanto con muy buen nivel. En la figura 3 podemos observar esta evolución.

Ciclos de Mejora en el Aula (2020). Experiencias de Innovación Docente de la US Esta obra se distribuye con la licencia Creative Commons 
PREGUNTA 1 INICIO

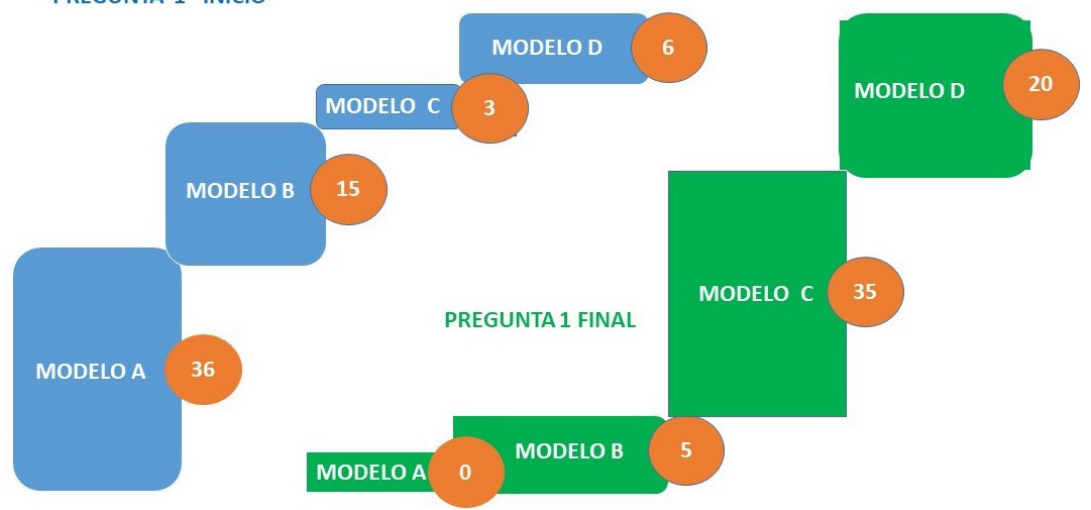

Figura 3. Escaleras de aprendizaje inicial y final de las preguntas: ¿Qué sabes acerca de la oficina de farmacia en España? ¿Funciona?

Tabla 6. Respuestas a las preguntas: ¿En base a qué 3 criterios se otorgan las aperturas? ¿Cómo se autoriza una farmacia?

\begin{tabular}{|c|c|c|}
\hline NIVELES DE CONOCIMIENTO & $\begin{array}{c}\text { CUESTIONARIO } \\
\text { INICIAL }\end{array}$ & $\begin{array}{c}\text { CUESTIONARIO } \\
\text { FINAL }\end{array}$ \\
\hline NIVEL A (No sé. No Sé). & $42(70 \%)$ & $0(0 \%)$ \\
\hline $\begin{array}{c}\text { NIVEL B (Habitantes y distancias. } \\
\text { No sé. / No sé. Instalación y/o } \\
\text { Funcionamiento) }\end{array}$ & $17(28,33 \%)$ & $6(10 \%)$ \\
\hline $\begin{array}{c}\text { NIVEL C (Farmacéutico propietario, y/o } \\
\text { habitantes y/o distancias. Instalación } \\
\text { y/o Funcionamiento). }\end{array}$ & $1(1,66 \%)$ & $17(28,33 \%)$ \\
\hline $\begin{array}{c}\text { NIVEL D (Farmacéutico propietario, } \\
\text { habitantes y distancias. Instalación y } \\
\text { Funcionamiento). }\end{array}$ & $0(0 \%)$ & $37(61,66 \%)$ \\
\hline
\end{tabular}

Los datos reflejan igualmente un cambio muy favorable pasando de algo más del $98 \%$ del nivel A/B en el cuestionario inicial; al $90 \%$ del nivel C/D en el cuestionario final. La comparativa de la figura 4 demuestra la inversión en los resultados.

Ciclos de Mejora en el Aula (2020). Experiencias de Innovación Docente de la US Esta obra se distribuye con la licencia Creative Commons 


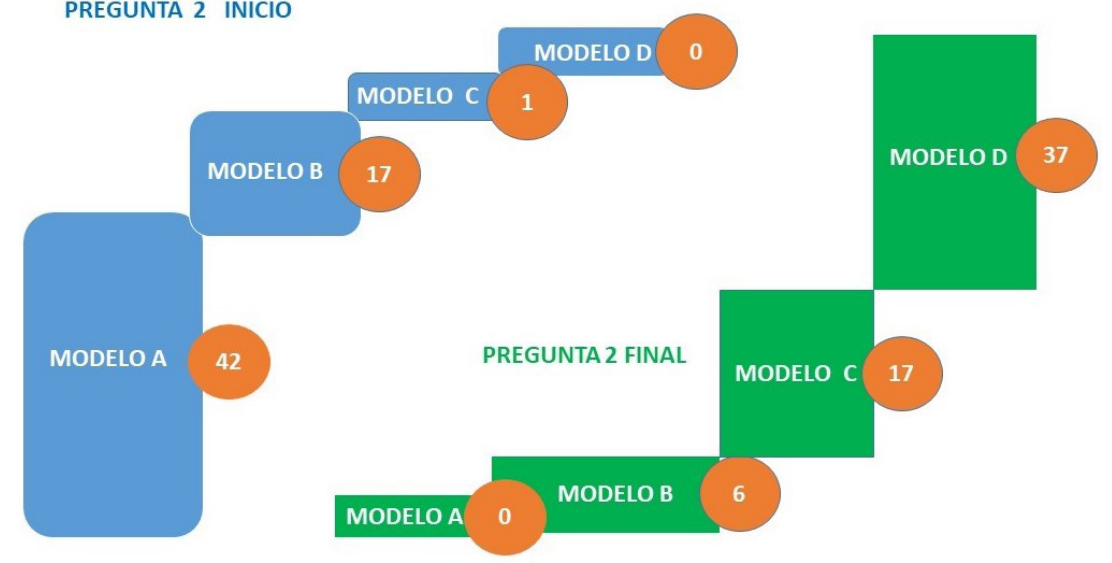

Figura 4. Escaleras de aprendizaje inicial y final a las preguntas: ¿En base a qué 3 criterios se otorgan las aperturas? ¿Cómo se autoriza una farmacia?

Tabla 7. Respuestas a las preguntas: ¿Se puede vender una farmacia? ¿Se puede heredar/donar una farmacia?

\begin{tabular}{|c|c|c|}
\hline $\begin{array}{c}\text { NIVELES DE } \\
\text { CONOCIMIENTO }\end{array}$ & CUESTIONARIO INICIAL & CUESTIONARIO FINAL \\
\hline NIVEL A (No. No) & $10(16,66 \%)$ & $0(0 \%)$ \\
\hline NIVEL B (Sí. No / No. Sí) & $8(13,33 \%)$ & $3(5 \%)$ \\
\hline NIVEL C (Sí. Sí) & $41(68,33 \%)$ & $30(50 \%)$ \\
\hline $\begin{array}{c}\text { NIVEL D (Sí. Sí. Con } \\
\text { regulaciones). }\end{array}$ & $1(1,66 \%)$ & $27(45 \%)$ \\
\hline
\end{tabular}

En esta sesión la mejora no ha sido tan espectacular ya que se refiere a un tema muy popular como es la compraventa y herencia de las farmacias por lo que el conocimiento previo C/D era del $70 \%$ que pasó al $95 \%$ al final del proceso. En la figura 4 enfrentamos las dos escaleras, Observamos que, aunque no es tan espectacular como en el resto, sí que ha resultado la sesión más debatida y que ha suscitado controversia, lo que ha permitido a cada postura defender y/o rebatir, y sobre todo repensar las ideas.

Ciclos de Mejora en el Aula (2020). Experiencias de Innovación Docente de la US Esta obra se distribuye con la licencia Creative Commons 


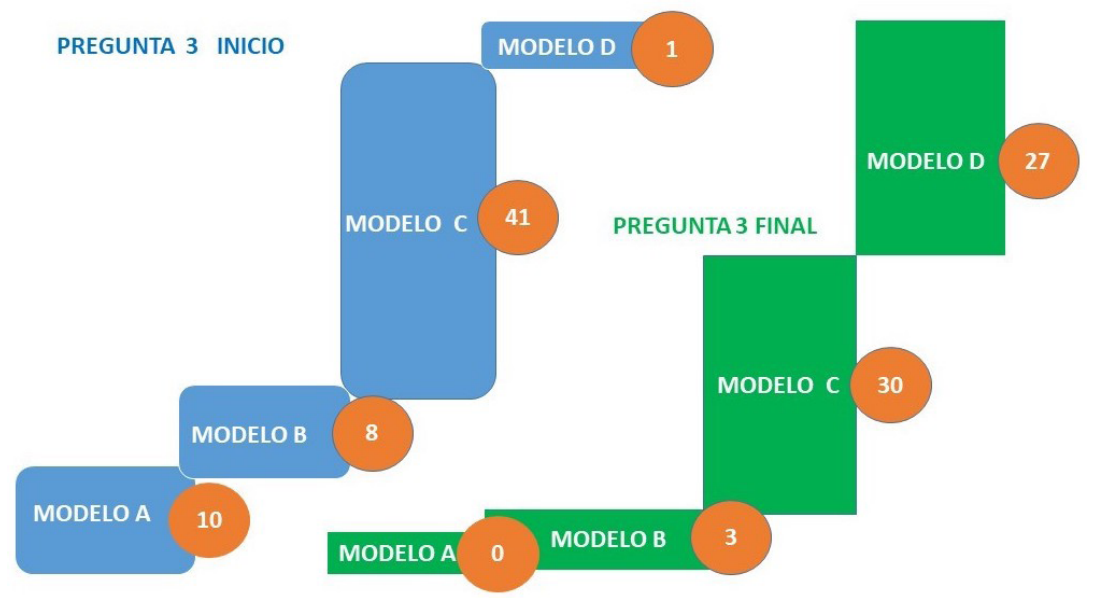

Figura 5. Escaleras de aprendizaje inicial y final de las preguntas: ¿Se puede vender una farmacia? ¿Se puede heredar/donar una farmacia?

Tabla 8. Respuestas a las preguntas ¿Se puede trasladar una farmacia? ¿Hay límites?

\begin{tabular}{|c|c|c|}
\hline $\begin{array}{c}\text { NIVELES DE } \\
\text { CONOCIMIENTO }\end{array}$ & CUESTIONARIO INICIAL & CUESTIONARIO FINAL \\
\hline NIVEL A (No. Sî) & $20(33,33 \%)$ & $0(0 \%)$ \\
\hline NIVEL B (Sí. No) & $23(38,33 \%)$ & $0(0 \%)$ \\
\hline NIVEL C (Sí. Sí) & $17(28,33 \%)$ & $45(75 \%)$ \\
\hline $\begin{array}{c}\text { NIVEL D (Sí. Sí. Puede } \\
\text { ser voluntario o } \\
\text { forzoso) }\end{array}$ & $0(0 \%)$ & $15(25 \%)$ \\
\hline
\end{tabular}

En esta sesión la mejora sí ha sido enorme ya que se refiere al traslado de farmacias donde había más lagunas. Los estudiantes en modelo/nivel A/B era superior al $70 \%$ al inicio; y al final del ciclo era de cero. El nivel C/D ha sido del $100 \%$ al terminar la sesión. Podemos ver en la figura 5 las escaleras de aprendizaje.

Ciclos de Mejora en el Aula (2020). Experiencias de Innovación Docente de la US Esta obra se distribuye con la licencia Creative Commons 


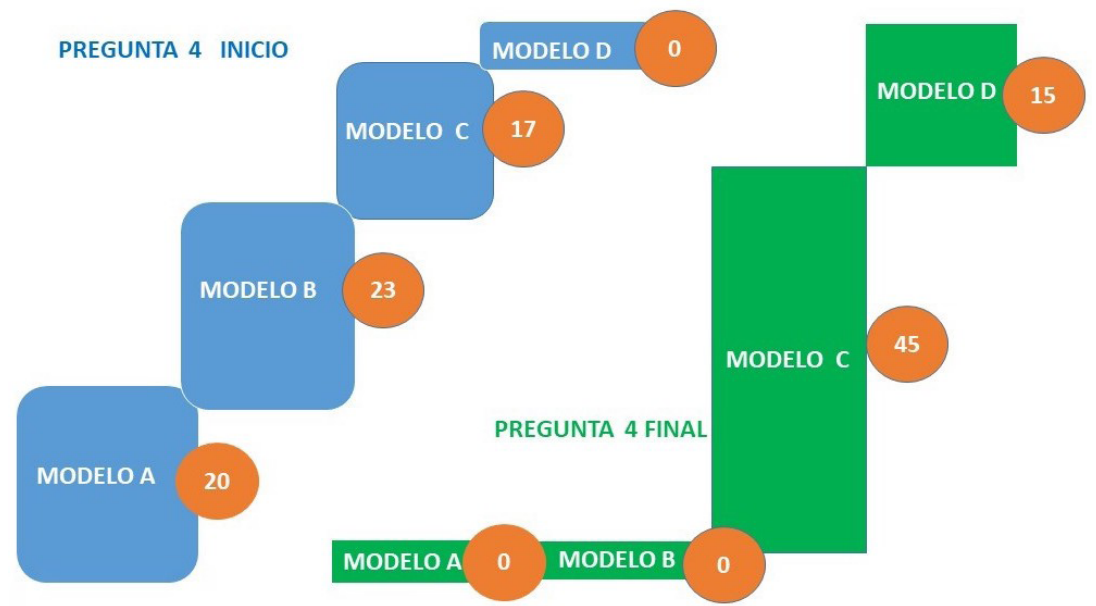

Figura 6. Escaleras de aprendizaje inicial y final de las preguntas: ¿Se puede trasladar una farmacia? ¿Hay límites?

Los contenidos de este CIMA son valorados en el examen parcial (30 de noviembre), y se verá incrementada la calificación por la participación de los estudiantes en el mismo.

En la clase del martes, 1 de diciembre, se pasó un test de valoración de la actividad usando la aplicación Wooclap de la plataforma de Blackboard Collaboration. Contestaron 51 estudiantes de los 60 que participaron en el CIMA.

Se realizaron dos preguntas cerradas, con los siguientes resultados:

1. Puntúa de 1 a 10, qué te ha parecido la actividad desarrollada con este formato de clases. Respuestas: 10 (22 estudiantes), 9 (19 estudiantes), 8 (8 estudiantes), 7 (2 estudiantes), resto (0 estudiantes).

2. ¿Qué aspecto (indicar uno solo) te ha gustado más de estas clases?

a) Los debates. (33 estudiantes)

b) La exposición de los compañeros. (2 estudiantes)

c) Los casos prácticos de las sentencias. (5 estudiantes)

d) Los videos. (1 estudiante)

Ciclos de Mejora en el Aula (2020). Experiencias de Innovación Docente de la US Esta obra se distribuye con la licencia Creative Commons 
e) La dinámica de participación colectiva. (10 estudiantes)

\section{Evaluación del ciclo de mejora docente}

Lo más importante, desde el punto de vista de personal, acorde a las lecturas realizadas (Porlán, 2017), es que aprender no debe ser un proceso unidireccional. Contenidos y profesor pueden ser sustituidos por otros contenidos y por los estudiantes. Es muy interesante cambiar el rol como profesor, desde ser esencial a ser secundario. Y el resultado final es mucho más óptimo. El grado de satisfacción del resultado por parte de este profesor ha sido alto. La calificación global otorgada por los estudiantes a esta actividad participativa del CIMA ha sido de una media de 9,20 sobre diez puntos. Lo que más les ha gustado han sido los debates (un 64,71\%) seguido de la participación colectiva (19,61\%). En definitiva, los estudiantes valoran las clases con una dinámica en la que ellos están plenamente implicados (más del 85\%).

El profesor solo debe ser guía cuando un debate se desvie o entre en punto muerto. También cuando se deban priorizar unos contenidos sobre otros. El empleo de la docencia invertida ha sido una experiencia muy enriquecedora que va a seguir manteniéndose en el futuro.

Lo más importante es que los contenidos han sido cuestionados, analizados y debatidos. Porque a veces es mejor abordar pocos temas, pero esenciales, en profundidad; que muchos, de modo superficial y frío. Y esta cuestión es una reflexión importante para el profesor. En cada aplicación del CIMA hemos ido anotando aquellas posibles mejoras. Una vez programado tenemos un esquema real de las actividades que nos reflejan aquellas materias de mayor interés. Ello nos plantea que debemos modificar para el futuro los tiempos dedicados en cada sesión a determinadas cuestiones, incrementando las más interesantes para ellos. Las preguntas de los estudiantes fueron Ciclos de Mejora en el Aula (2020). Experiencias de Innovación Docente de la US
Esta obra se distribuye con la licencia Creative Commons 
respondidas por aquellos compañeros que habían trabajado cada sesión. Se anotaron esas preguntas y se plantea la posibilidad de utilizar algunas de ellas como preguntas/ problemas en el futuro cuestionario. Todas estas cuestiones/detalles se han ido anotando en un diario de sesiones.

La comparativa de las escaleras de aprendizaje, tras los cuestionarios inicial y final, nos han ayudado a cotejar qué temáticas son mejor conocidas, aprendidas y cuáles no. Y a partir de ello, intentaremos subsanarlas en el siguiente CIMA.

En resumen, el curso y las aplicaciones de los dos $\mathrm{Cl}-$ MAs nos han servido para plantear alternativas a la clase magistral y que ésta vaya siendo cada vez menor en el porcentaje del contenido de la asignatura.

Ciclos de Mejora en el Aula (2020). Experiencias de Innovación Docente de la US Esta obra se distribuye con la licencia Creative Commons 
Palabras clave: Legislación farmacéutica, Farmacia, docencia universitaria, experimentación docente universitaria, debate.

Keywords: Pharmaceutical law, Pharmacy, university teaching, university teaching experimentation, debate.

\section{Referencias Bibliográficas}

Bain, K. (2007). Lo que hacen los mejores profesores universitarios. Valencia: Universitat de Valencia. Servei de Publicacions.

Finkel, D. (2008). Dar clase con la boca cerrada. Valencia: Universitat de Valencia. Servei de Publicacions.

Porlán, R. (2017). Enseñanza universitaria. Cómo mejorarla. Madrid: Ediciones Morata.

Ciclos de Mejora en el Aula (2020). Experiencias de Innovación Docente de la US Esta obra se distribuye con la licencia Creative Commons 